https://helda.helsinki.fi

\title{
L1CAM expression associates with poor outcome in endometrioid, but not in clear cell ovarian carcinoma
}

Soovares, Piret

2017-09

Soovares , P , Pasanen , A , Butzow , R \& Lassus , H 2017 , ' L1CAM expression associates with poor outcome in endometrioid, but not in clear cell ovarian carcinoma ', Gynecologic Oncology , vol. 146 , no. 3 , pp. 615-622 . https://doi.org/10.1016/j.ygyno.2017.06.010

http://hdl.handle.net/10138/297948

https://doi.org/10.1016/j.ygyno.2017.06.010

publishedVersion

Downloaded from Helda, University of Helsinki institutional repository.

This is an electronic reprint of the original article.

This reprint may differ from the original in pagination and typographic detail.

Please cite the original version. 


\title{
L1CAM expression associates with poor outcome in endometrioid, but not in clear cell ovarian carcinoma
}

\author{
Piret Soovares ${ }^{\mathrm{a}}$, Annukka Pasanen ${ }^{\mathrm{b}}$, Ralf Bützow ${ }^{\mathrm{b}}$, Heini Lassus ${ }^{\mathrm{c}, *}$ \\ a Department of Obstetrics and Gynecology, University of Helsinki, Helsinki University Hospital, Haartmaninkatu 2, PO Box 140, 00290 Helsinki, Finland \\ ${ }^{\mathrm{b}}$ Department of Pathology, University of Helsinki, Helsinki University Hospital, Haartmaninkatu 3, 00290 Helsinki, Finland \\ c Department of Obstetrics and Gynecology, University of Helsinki, Helsinki University Hospital, Haartmaninkatu 2, PO Box 140, 00290 Helsinki, Finland
}

\section{H I G H L I G H T S}

- L1CAM predicts poor outcome in endometrioid, but not in clear cell ovarian carcinoma.

- Our findings are similar to those previously observed in endometrial carcinoma.

- L1CAM associates with poor outcome in grade 1-2 endometrioid ovarian carcinoma.

- L1CAM predicts poor prognosis in concurrent ovarian and endometrial carcinomas.

\section{A R T I C L E I N F O}

\section{Article history:}

Received 2 March 2017

Received in revised form 2 June 2017

Accepted 7 June 2017

Available online 16 June 2017

\section{Keywords:}

Ovarian cancer

L1CAM

Endometrioid

Clear cell

Prognosis

Response to therapy

\begin{abstract}
A B S T R A C T
Objective. Our aim was to study the expression of L1CAM in endometrioid and clear cell ovarian carcinomas and to evaluate its correlation with clinical parameters and patient prognosis.

Methods. Tissue microarray -based immunohistochemical analysis of L1CAM expression was performed in 249 endometrioid and 140 clear cell ovarian carcinomas. Concurrent endometrial carcinoma was found in 57 of these patients.
\end{abstract}

Results. L1CAM expression was found in 15\% of endometrioid and 23\% of clear cell ovarian carcinomas. L1CAM expression was strongly associated with poor disease-specific overall survival and poor disease-free survival in endometrioid ( $p<0.0001, p=0.0005)$, but not in clear cell ovarian carcinomas. Significant association of L1CAM expression with poor overall survival was observed in grade $1-2$ carcinomas $(p<0.0001)$, but not in grade 3 tumors. In endometrioid ovarian carcinomas, L1CAM expression was associated with aggressive tumor characteristics, such as higher grade and stage, and incomplete response to primary therapy. However, L1CAM expression was not an independent prognostic factor for overall or disease-free survival. Of the 57 patients with concurrent endometrial carcinoma L1CAM positivity was found in 4 cases both in the ovarian and endometrial tumors, and in 3 cases only in the endometrial tumor. All these seven patients with L1CAM positive tumors had poor outcome.

Conclusions. L1CAM expression could serve as a biomarker for predicting clinical outcome and response to therapy in patients with endometrioid ovarian carcinoma, but not in clear cell carcinomas. L1CAM positivity also predicts poor outcome in patients with concurrent endometrioid ovarian and endometrial carcinomas.

(C) 2017 Elsevier Inc. All rights reserved.

\section{Introduction}

Ovarian cancer is the seventh most common cancer in women and a primary cause of death from gynecologic cancers [1]. Late diagnosis and acquired platinum resistance often result in poor overall prognosis [2]. Ovarian carcinomas are histologically divided into serous, endometrioid,

\footnotetext{
* Corresponding author.

E-mail addresses: piret.soovares@hus.fi (P. Soovares), annukka.pasanen@hus.fi (A. Pasanen), ralf.butzow@hus.fi (R. Bützow), heini.lassus@hus.fi (H. Lassus).
}

clear cell, mucinous, undifferentiated, mixed and Brenner subtypes [3]. A dualistic model of ovarian carcinogenesis has been proposed: type I carcinomas being more indolent and often confined to the ovary resulting in good prognosis and type II tumors being more aggressive and accounting for $90 \%$ of the deaths from ovarian cancer. Type II tumors include high grade serous and undifferentiated carcinomas as well as carcinosarcomas. Other histological types belong to type I tumors. However, molecular studies have shown that there is heterogeneity within different types of ovarian carcinoma. Tumors with different biology, clinical behavior and prognosis can be found [4]. 
Currently, the conventional treatment of ovarian carcinomas includes staging/debulking surgery and adjuvant platinum-taxane based chemotherapy for all cases regardless of the tumor type (except no chemotherapy in stage IA grade 1 disease) [2]. Of the novel targeted therapies, bevacizumab and olaparib have been approved for treatment of ovarian cancer. Olaparib is the first drug with a predictive marker (BRCA mutation), which selects patients who are likely to have the greatest benefit of the treatment [5]. To individualize treatment of the ovarian cancer patients, we need more biomarkers for detecting tumors with different biological behavior and to select patients for novel targeted therapies.

L1CAM (CD171) is a cell adhesion molecule, a transmembrane glycoprotein of 200-220 kDda, which belongs to the immunoglobulin superfamily. L1CAM is involved in myelination, fasciculation, cell migration and axon guidance, thus playing an important role in the development of the nervous system [6-8]. L1CAM overexpression has been found in several cancers as well: e.g. ovarian [9-11], endometrial [9,12-16], colorectal $[17,18]$, gastric $[19,20]$ and breast cancer $[21,22]$.

In endometrial carcinomas L1CAM positivity has been associated with aggressive disease characteristics, such as advanced stage, poor differentiation, non-endometrioid histology, lymph node involvement, lymphovascular space invasion, cervical stromal invasion, positive peritoneal cytology and distant recurrences $[9,12,13,15,16]$. In endometrial carcinomas L1CAM expression predicts poor outcome particularly in the endometrioid but not in the serous or clear cell subtypes $[15,16]$. Thus L1CAM is a potential marker of high risk disease in stage I endometrioid endometrial carcinomas which are considered clinically as low risk carcinomas.

Endometrioid endometrial and ovarian carcinomas are histologically similar and they are known to share some pathogenetic changes like mutations of PTEN and $\beta$-catenin genes as well as microsatellite instability $[4,23]$. Clear cell carcinoma in turn resembles endometrioid ovarian carcinoma by sharing background of endometriosis and mutations of ARID1A and catenin $\beta 1$ as well as PI3K pathway activation [4]. Approximately one third of endometrioid and clear cell ovarian carcinomas have already spread at the time of the diagnosis and in these cases the prognosis is poor. The prognosis in stage I endometrioid and clear cell ovarian carcinomas is generally favorable. However, in some patients the disease relapses and the outcome is poor. New biomarkers are needed to better understand the pathogenesis of these diseases and distinguish groups with different biological behavior and prognosis.

In ovarian carcinomas, L1CAM expression and its clinical relevance has mainly been investigated in the most common subtype, high grade serous carcinoma [9,10,24-26], and only a limited number of endometrioid carcinomas have been studied [9-11]. Survival analyses and clinical correlations on the endometrioid type do not exist and expression of L1CAM in clear cell ovarian carcinomas has not been previously investigated.

Our study involves a large number of consecutive patients treated for endometrioid (249 patients) and clear cell (140 patients) ovarian carcinomas at the same institution. In 57 cases concurrent endometrial carcinoma was diagnosed. Our aim was to investigate the expression of L1CAM in these carcinomas and to evaluate its correlation with various clinical parameters and patient prognosis.

\section{Materials and methods}

\subsection{Patients}

The study included 249 patients treated for endometrioid ovarian carcinoma and 140 patients treated for clear cell ovarian carcinoma at the Department of Obstetrics and Gynecology of the Helsinki University Central Hospital between January 1, 1989 and December 31, 2013.

Consecutive patients treated for endometrioid and clear cell ovarian carcinoma were searched according to pathological records. Approval from the Ethics Committee was obtained.
A gynecological pathologist had determined the histology of those carcinomas at the time of diagnosis. Histological diagnoses were reviewed by a gynecological pathologist before including cases in the study. Clinical data of the patients was collected from the hospital records, and additional survival information was received from the Population Register Center.

All the patients included in the study underwent surgery. Median age at surgery was 59.0 years in the endometrioid ovarian carcinoma group and 58.0 years in the clear cell carcinoma group. Our institution is a tertiary hospital, where treatment of ovarian carcinoma is centralized. Radical surgery was started at the end of the 1980's. In 278 of the 389 patients total abdominal or laparoscopic hysterectomy, bilateral salpingo-oophorectomy, omentectomy as well as pelvic and/or para-aortic lymphadenectomy were performed. In 73 patients, total abdominal or laparoscopic hysterectomy and bilateral salpingo-oophorectomy (and omentectomy) were performed in conjunction with surgical removal of tumor masses. In 38 patients, uni-or bilateral salpingo-oophorectomy or explorative laparoscopy was performed. In most of the cases where lymphadenectomy was not performed, there was presence of carcinosis/advanced disease. The rate of lymphadenectomy increased a little over the study period: it was $67.9 \%$ before year 2000 and $74.5 \%$ after year 2000 . In the beginning of the study period, the optimal debulking was regarded as $<2 \mathrm{~cm}$ of residual tumor. Gradually the optimal debulking goal decreased to no residual tumor. To minimize bias in this study, we used the limit of $0 \mathrm{~cm}$ for optimal surgery for the whole study period. The information was extracted from the operation records. The tumor specimens for the study were obtained from the primary surgery.

In 52 endometrioid ovarian carcinoma patients and 5 clear cell ovarian carcinoma patients concurrent endometrial carcinoma was diagnosed. In all endometrioid ovarian carcinoma cases, the concurrent endometrial carcinoma was of endometrioid type. In three of the clear cell ovarian carcinoma cases, the concurrent endometrial carcinoma was of clear cell type and in two cases of endometrioid type. The staging of tumors was performed according to the year 2009 FIGO staging system. Grading was applied only to the endometrioid carcinomas.

In 256 cases platinum-taxane-based combination therapy was given as first-line chemotherapy, and in 7 of those cases bevacizumab was added to the treatment. In 5 cases, the patient received platinum plus other than taxane-based chemotherapy. In 29 cases, the patient received carboplatin as single therapy. Other than platinum-based chemotherapy was administered to 40 patients (in the late 1980s and in the beginning of the 1990s). In 55 cases no adjuvant chemotherapy was given. Most of the patients who did not receive chemotherapy had stage I A grade 1 endometrioid ovarian carcinoma. A few patients had low performance status or high age or refused the treatment.

Response to therapy was assessed after the initial 6-8 cycles of chemotherapy on the basis of gynecological examination, vaginal ultrasonography, CA125 measurement, and/or computed tomography scan. In the late 1980s and in the beginning of the 1990s second look laparotomy was performed routinely. Patients who did not receive chemotherapy were evaluated 5-6 months after the surgery. Disease-specific overall survival was calculated from the date of diagnosis (primary surgery) to death from ovarian carcinoma. Patients who died of other causes or were alive at follow-up were censored. Disease-free survival was calculated for patients who presented with complete response after the primary treatment (surgery and first-line chemotherapy, if given) and it was the time from the date of diagnosis to relapse of the disease. The median follow-up time for patients who were cencored at the end of the follow-up was 7.8 years (range $0.75-23.6$ years) for endometrioid ovarian carcinoma and 9.3 years (range 1.7-25.3 years) for clear cell ovarian carcinoma.

\subsection{Tissue microarray construction}

Histological slides were examined by a gynecological pathologist and representative areas of each tumor were selected for biopsies. Four 
duplicate $0.8 \mathrm{~mm}$ cores were drawn from the corresponding area of the paraffin blocks and TMA blocks were prepared.

\subsection{Immunohistochemistry}

Immunohistochemical staining protocols were carried out with Ventana Benchmark XT automated slide preparation system (Ventana Medical Systems, Inc., USA). Briefly, slides were deparaffinized and heat-induced epitope retrieval was performed in CC1 solution following standard protocol. Tissue sections were incubated with primary monoclonal antibodies against L1CAM (CD171, Covance, clone 14.10, catalog number SIG-3911-1000, dilution $1: 100$ for $60 \mathrm{~min}$ ). The antibody binding site was visualized using UltraView Universal DAB Detection Kit. Sections were counterstained with DAKO Mayer's hematoxylin (S3309), dehydrated, cleared in xylene, and mounted (Fig. 1).

Slides were scored by two authors (P.S., A.P.) blinded to clinical data. Equivocal cases were re-evaluated and a consensus score was formed. Membranous L1CAM staining of cancer cells was scored as previously described [16]: cases were defined L1CAM positive when membranous staining of $>10 \%$ of cancer cells was present.

The accuracy of a tissue microarray (TMA)-based immunohistochemical analysis largely depends on the uniformity of the staining pattern for a given antigen and the number of biopsy cores included in the TMA. We have previously demonstrated a good concordance rate ( $\kappa$ value 0.835 ) between L1CAM staining results of a 4-core TMA and the corresponding whole sections in endometrial carcinoma [16]. To further address this issue, we analyzed L1CAM staining in whole tissue slides of 12 ovarian tumors ( 6 endometrioid and 6 clear cell carcinomas) that were L1CAM positive on the TMA. The heterogeneous staining pattern seen on ovarian sections was identical to that seen in endometrial carcinoma and the concordance rate between our 4-core TMA and whole slides was perfect (100\%).

\subsection{Statistical analysis}

Data regarding endometrioid and clear cell ovarian carcinomas were analyzed separately. Categorical variables were compared with Pearson $\chi^{2}$ test and Fisher's exact test. Bonferroni correction was used for correction of multiple variable testing (association of L1CAM with clinicopathological characteristics). Bonferroni correction set statistical significance at $<0.0063$ for endometrioid carcinomas (eight clinicopathological factors) and $<0.0071$ for clear cell ovarian carcinoma (seven factors, grade not applicable). The disease-specific overall survival and disease-free survival was estimated using the Kaplan-Meier method and differences between groups were compared with the log rank test. For multivariate survival analysis Cox proportional hazards model was used with the following covariates: FIGO stage (I-II vs. III-IV), grade (1-2 vs. 3), tumor size $(\leq 10 \mathrm{~cm}$ vs. $>10 \mathrm{~cm})$, residual tumor (presence vs. absence), L1CAM expression (negative vs. positive), ascites in general (presence vs. absence) and age. Statistical significance was set at $p<0.05$. Data was analyzed using IBM SPSS version 22 software.

\section{Results}

L1CAM staining was not interpretable in 17 (4.3\%) of the 389 carcinomas due to lack of tumor tissue, loss of biopsy specimens, or staining artifacts.
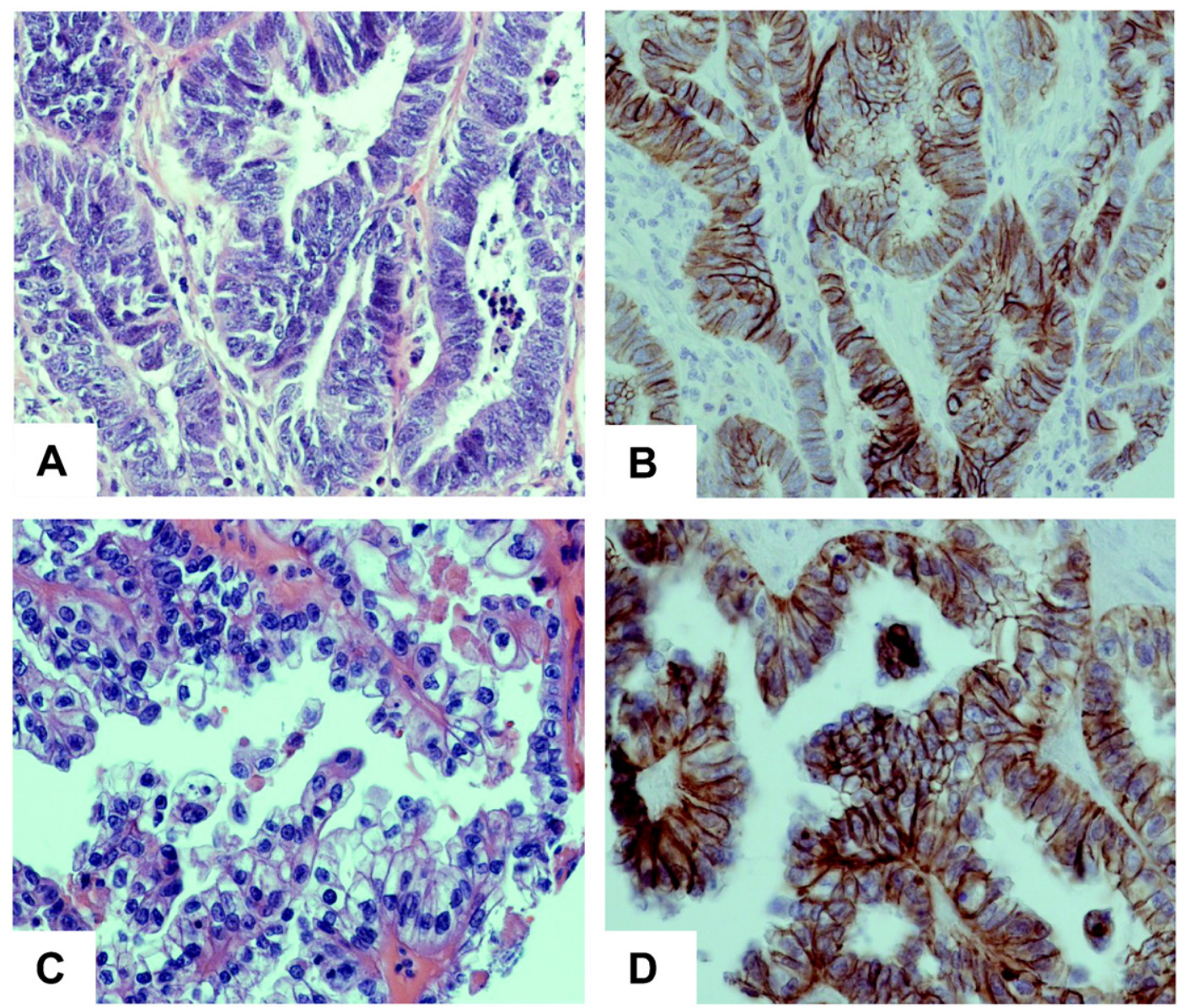

Fig. 1. Hematoxylin and eosin and immunohistochemical staining for L1CAM in TMA sections of endometrioid (A,B) and clear cell (C,D) ovarian carcinoma. 


\subsection{Clinicopathological characteristics}

\subsubsection{Endometrioid ovarian carcinomas}

L1CAM positivity was detected in 36 (15\%) of the 235 interpretable endometrioid ovarian carcinomas. There was no significant difference between L1CAM-positive and -negative carcinomas regarding tumor size, ascites, CA125-level or concurrent endometrial carcinoma. L1CAM expression was significantly associated with clinicopathological characteristics such as greater patient age, higher grade and stage as well as presence of residual tumor in primary surgery (Table 1). When Bonferroni correction was applied to the analyses, there was significant association of positive L1CAM with high grade and stage. Only $5 \%$ of grade 1 carcinomas were L1CAM-positive, whereas the percentage was 33 in the grade 3 group. L1CAM-positivity was found in 7\% of stage I-II and $28 \%$ of stage III-IV carcinomas. Of the patients with no residual tumor in the primary surgery, $11 \%$, and of those with the presence of residual tumor, 26\% were L1CAM-positive.

Positive L1CAM was associated with incomplete response to primary therapy ( $p=0.02$, Table 1 ). L1CAM-positivity was found in $13 \%$ of those who had a complete response to primary therapy, whereas the percentage was 27 in those with partial response, stable or progressive disease.

\subsubsection{Clear cell ovarian carcinomas}

Positive L1CAM was detected in $32(23 \%)$ of the 137 interpretable clear cell ovarian carcinomas. L1CAM expression was associated with larger tumor size: $12 \%$ of the tumors smaller than $10 \mathrm{~cm}$ were L1CAMpositive, whereas $28 \%$ of the tumors larger than $10 \mathrm{~cm}$ were L1CAMpositive. Otherwise there was no difference regarding clinicopathological characteristics between L1CAM-negative and L1CAM-positive tumors (Table 2). L1CAM-positivity was found in $20 \%$ of stage I-II carcinomas

Table 1

Clinicopathological characteristics, response to therapy and L1CAM expression in patients with endometrioid ovarian carcinoma. CR, complete remission; PR, partial remission; SD, stable disease; PD, progressive disease.

\begin{tabular}{|c|c|c|c|c|}
\hline & Total & L1CAM negative & L1CAM positive & $p$-Value \\
\hline No. of patients & 235 & 199 (85\%) & $36(15 \%)$ & \\
\hline $\begin{array}{l}\text { Age at surgery } \\
<59 \text { years (median) } \\
\geq 59 \text { years (median) }\end{array}$ & $\begin{array}{l}115(49 \%) \\
120(51 \%)\end{array}$ & $\begin{array}{l}103(52 \%) \\
96(48 \%)\end{array}$ & $\begin{array}{l}12(33 \%) \\
24(67 \%)\end{array}$ & 0.04 \\
\hline $\begin{array}{l}\text { Histological grade } \\
1 \\
2 \\
3\end{array}$ & $\begin{array}{l}108(46 \%) \\
79(34 \%) \\
48(20 \%)\end{array}$ & $\begin{array}{l}103(52 \%) \\
64(32 \%) \\
32(16 \%)\end{array}$ & $\begin{array}{l}5(14 \%) \\
15(42 \%) \\
16(44 \%)\end{array}$ & $<0.0001$ \\
\hline $\begin{array}{l}\text { Disease stage } \\
\text { I-II } \\
\text { III-IV }\end{array}$ & $\begin{array}{l}147(63 \%) \\
86(37 \%)\end{array}$ & $\begin{array}{l}136(69 \%) \\
62(31 \%)\end{array}$ & $\begin{array}{l}11(31 \%) \\
24(69 \%)\end{array}$ & $<0.0001$ \\
\hline $\begin{array}{l}\text { Tumor size } \\
\leq 10 \mathrm{~cm} \\
>10 \mathrm{~cm}\end{array}$ & $\begin{array}{l}102(44 \%) \\
132(56 \%)\end{array}$ & $\begin{array}{l}85(43 \%) \\
113(57 \%)\end{array}$ & $\begin{array}{l}17(47 \%) \\
19(53 \%)\end{array}$ & 0.63 \\
\hline $\begin{array}{l}\text { Ascites } \\
\text { No } \\
\text { Yes }\end{array}$ & $\begin{array}{l}83(36 \%) \\
149(64 \%)\end{array}$ & $\begin{array}{l}69(35 \%) \\
127(65 \%)\end{array}$ & $\begin{array}{l}14(39 \%) \\
22(61 \%)\end{array}$ & 0.67 \\
\hline $\begin{array}{l}\text { Residual tumor } \\
\text { No } \\
\text { Yes }\end{array}$ & $\begin{array}{l}171(75 \%) \\
58(25 \%)\end{array}$ & $\begin{array}{l}152(78 \%) \\
43(22 \%)\end{array}$ & $\begin{array}{l}19(56 \%) \\
15(44 \%)\end{array}$ & 0.006 \\
\hline $\begin{array}{l}\text { CA125 } \\
\text { Normal } \\
\text { Elevated }\end{array}$ & $\begin{array}{l}40(17 \%) \\
190(83 \%)\end{array}$ & $\begin{array}{l}34(18 \%) \\
160(82 \%)\end{array}$ & $\begin{array}{l}6(17 \%) \\
30(83 \%)\end{array}$ & 0.90 \\
\hline $\begin{array}{l}\text { Concurrent endomet } \\
\text { No } \\
\text { Yes }\end{array}$ & $\begin{array}{l}\text { ial carcinoma } \\
186(79 \%) \\
49(21 \%)\end{array}$ & $\begin{array}{l}155(78 \%) \\
44(22 \%)\end{array}$ & $\begin{array}{l}31(86 \%) \\
5(14 \%)\end{array}$ & 0.26 \\
\hline $\begin{array}{l}\text { Response to therapy } \\
\text { CR } \\
\text { PR, SD or PD }\end{array}$ & $\begin{array}{l}183(79 \%) \\
49(21 \%)\end{array}$ & $\begin{array}{l}160(82 \%) \\
36(18 \%)\end{array}$ & $\begin{array}{l}23(64 \%) \\
13(36 \%)\end{array}$ & 0.02 \\
\hline
\end{tabular}

and in $28 \%$ of stage III-IV carcinomas. When Bonferroni correction was applied to the analyses, the association on positive L1CAM with larger tumor size was not statistically significant.

L1CAM expression was not associated with response to primary therapy ( $p=0.72$, Table 2 ). L1CAM-positivity was found in $23 \%$ of those who had a complete response to primary therapy, whereas the percentage was $26 \%$ in those with partial response, stable or progressive disease.

\subsection{Disease-specific overall survival and disease-free survival}

\subsubsection{Endometrioid ovarian carcinomas}

In endometrioid ovarian carcinomas, positive L1CAM expression was strongly associated with poor overall survival $(p<0.0001)$ (Fig. 2A). The 5 -year overall survival rate was $71 \%$ for the whole cohort, $46 \%(95 \% \mathrm{CI}$, $29-62 \%)$ for the L1CAM-positive group and $76 \%(70-82 \%)$ for the L1CAM-negative group.

We also assessed the association of L1CAM status with relapse after primary treatment. Patients with L1CAM-positive endometrioid ovarian carcinomas had shorter disease-free survival time than patients with L1CAM-negative carcinomas ( $p=0.0005$ ) (Fig. 2B). The 5-year diseasefree survival rate was $75 \%$ for the whole group, $52 \%$ (32-73\%) for the L1CAM-positive group and 80\% (74-87\%) for the L1CAM-negative group.

As paclitaxel was not used in the beginning of the study period, we analyzed separately only patients treated with paclitaxel-platinum based chemotherapy and positive L1CAM expression was still associated with poor overall and disease-free survival $(p=0.0086$ and $p=0.0044$ respectively). (Sup. Fig. 1A-B). As surgery has become more radical over the study period, we also analyzed separately patients treated starting from the year 2000 and found that L1CAM was still significantly associated with poor overall and disease-free survival $(p=0.002$ and $p=0.001$ respectively; Sup. Fig. 1C-D).

Clinically interesting subgroups were analyzed separately for overall survival. Significant association of L1CAM expression with poor overall survival was observed in grade $1-2$ carcinomas $(p<0.0001)$, but no

Table 2

Clinicopathological characteristics, response to therapy and L1CAM expression in patients with clear cell ovarian carcinoma. CR, complete remission; PR, partial remission; SD, stable disease; PD, progressive disease.

\begin{tabular}{|c|c|c|c|c|}
\hline & Total & L1CAM negative & L1CAM positive & $p$-Value \\
\hline No. of patients & 137 & 105 (77\%) & $32(23 \%)$ & \\
\hline $\begin{array}{l}\text { Age at surgery } \\
<58 \text { years (median) } \\
\geq 58 \text { years (median) }\end{array}$ & $\begin{array}{l}65(47 \%) \\
72(53 \%)\end{array}$ & $\begin{array}{l}52(50 \%) \\
53(50 \%)\end{array}$ & $\begin{array}{l}13(41 \%) \\
19(59 \%)\end{array}$ & 0.37 \\
\hline $\begin{array}{l}\text { Disease stage } \\
\text { I-II } \\
\text { III-IV }\end{array}$ & $\begin{array}{l}83(61 \%) \\
54(39 \%)\end{array}$ & $\begin{array}{l}66(63 \%) \\
39(37 \%)\end{array}$ & $\begin{array}{l}17(53 \%) \\
15(47 \%)\end{array}$ & 0.32 \\
\hline $\begin{array}{l}\text { Tumor size } \\
\leq 10 \mathrm{~cm} \\
>10 \mathrm{~cm}\end{array}$ & $\begin{array}{l}42(31 \%) \\
92(69 \%)\end{array}$ & $\begin{array}{l}37(36 \%) \\
66(64 \%)\end{array}$ & $\begin{array}{l}5(16 \%) \\
26(84 \%)\end{array}$ & 0.04 \\
\hline $\begin{array}{l}\text { Ascites } \\
\text { No } \\
\text { Yes }\end{array}$ & $\begin{array}{l}52(39 \%) \\
82(61 \%)\end{array}$ & $\begin{array}{l}42(41 \%) \\
61(59 \%)\end{array}$ & $\begin{array}{l}10(32 \%) \\
21(68 \%)\end{array}$ & 0.39 \\
\hline $\begin{array}{l}\text { Residual tumor } \\
\text { No } \\
\text { Yes }\end{array}$ & $\begin{array}{l}93(69 \%) \\
42(31 \%)\end{array}$ & $\begin{array}{l}75(73 \%) \\
28(27 \%)\end{array}$ & $\begin{array}{l}18(56 \%) \\
14(44 \%)\end{array}$ & 0.08 \\
\hline $\begin{array}{l}\text { CA125 } \\
\text { Normal } \\
\text { Elevated }\end{array}$ & $\begin{array}{l}30(23 \%) \\
100(77 \%)\end{array}$ & $\begin{array}{l}25(25 \%) \\
76(75 \%)\end{array}$ & $\begin{array}{l}5(17 \%) \\
24(83 \%)\end{array}$ & 0.40 \\
\hline $\begin{array}{l}\text { Concurrent endomet } \\
\text { No } \\
\text { Yes }\end{array}$ & $\begin{array}{l}\text { ial carcinom } \\
132(96 \%) \\
5(4 \%)\end{array}$ & $\begin{array}{l}100(95 \%) \\
5(5 \%)\end{array}$ & $\begin{array}{l}32(100 \%) \\
0(0 \%)\end{array}$ & 0.60 \\
\hline $\begin{array}{l}\text { Response to therapy } \\
\text { CR } \\
\text { PR, SD or PD }\end{array}$ & $\begin{array}{l}101(74 \%) \\
35(26 \%)\end{array}$ & $\begin{array}{l}78(75 \%) \\
26(25 \%)\end{array}$ & $\begin{array}{l}23(72 \%) \\
9(28 \%)\end{array}$ & 0.72 \\
\hline
\end{tabular}



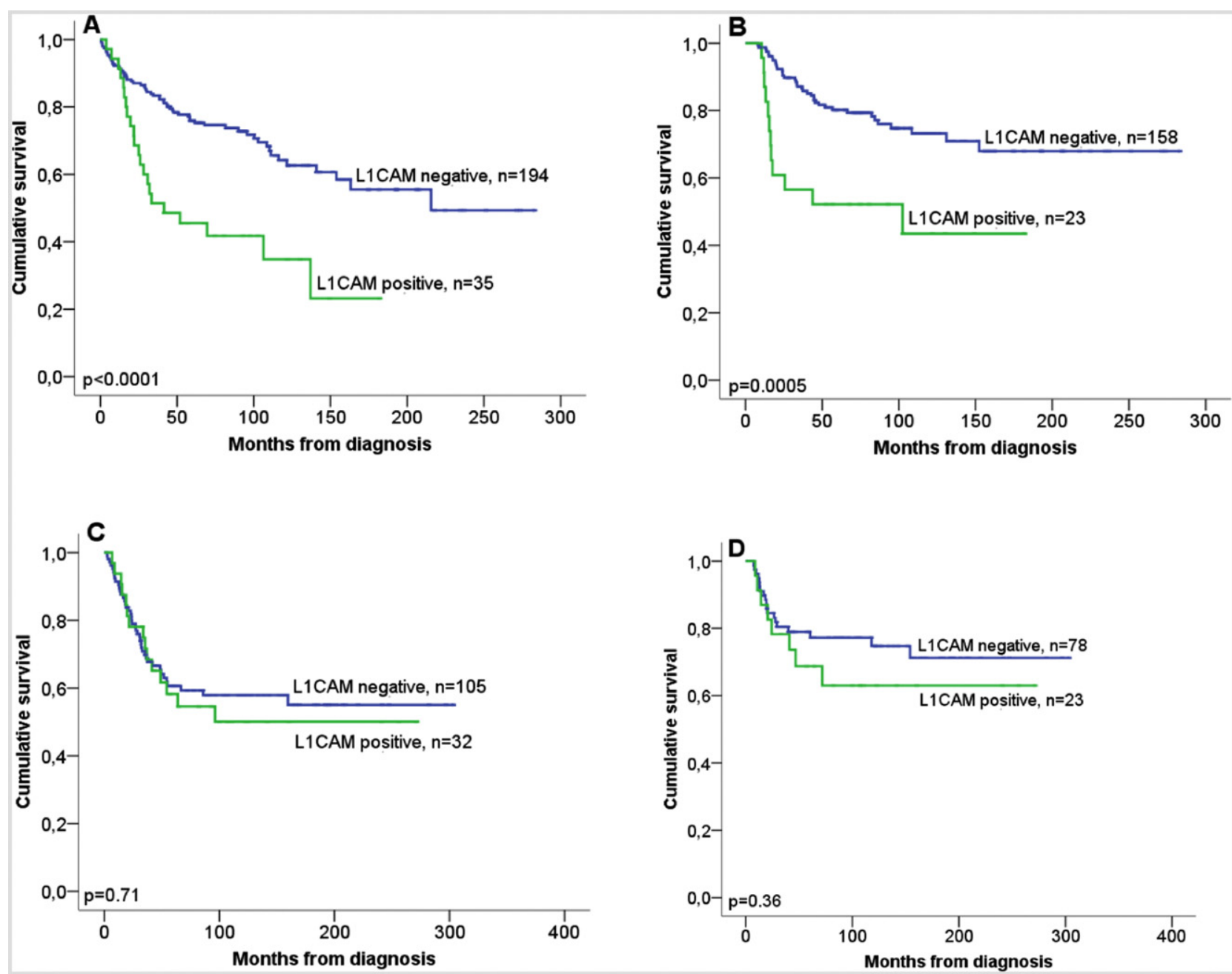

Fig. 2. Disease-specific overall and disease-free survival in patients with endometrioid ovarian carcinoma (A,B) and clear cell ovarian carcinoma (C,D).

association was found in grade 3 tumors (Fig. 3A-B). In stage I tumors there was no difference in overall survival between L1CAM positive and negative cases. In stage III carcinomas, there was a tendency for poorer overall survival for L1CAM-positive cases, but there was no statistically significant difference between the groups $(p=0.15)$ (Fig. 3C-D).

In multivariate analysis, L1CAM expression was not an independent prognostic factor for overall or disease free survival. Stage and the presence of residual tumor in primary surgery were independent prognostic factors for overall survival ( $p<0.0001$ and $p=0.003$ respectively). Stage and grade were independent prognostic factors for disease free survival $(p<0.0001$ and $p=0.007$ respectively $)$.

\subsubsection{Clear cell ovarian carcinomas}

L1CAM expression was not associated with shorter overall survival in clear cell ovarian carcinomas ( $p=0.71$ ). The 5 -year overall survival rate was $61 \%$ for the whole group, $58 \%$ ( $41-76 \%$ ) for the L1CAM positive group and 61\% (51-70\%) for the L1CAM negative group (Fig. 2C).

There was no significant difference in disease-free survival between L1CAM-negative and L1CAM-positive groups $(p=0.36)$. The 5-year disease-free survival rate was $77 \%$ for the whole group, $69 \%$ (49-88\%) for the L1CAM-positive group and 79\% (70-88\%) for the L1CAM-negative group (Fig. 2D).

\subsection{Disease recurrences in endometrioid ovarian carcinomas}

Complete response to primary treatment was found in 196 (79\%) of the 249 patients with endometrioid ovarian carcinoma. Of these, in 55 patients (28\%) the disease relapsed. Of the patients who had a recurrence, 12 (22\%) had L1CAM-positive tumors and 38 (69\%) had L1CAMnegative tumors. In 5 (9\%) patients the L1CAM-status was not interpretable.

Relapses in the pelvis were equally common in L1CAM-positive and L1CAM-negative cases (40\% vs. $47 \%$ ). Relapses in pelvic or para-aortic lymph nodes were more common in L1CAM-positive than L1CAM-negative carcinomas (27\% vs. $6 \%$ ). Distant relapses were less frequent in L1CAM-positive than in L1CAM-negative cases (30\% vs. 46\%). However, the numbers of cases in each group were fairly small and the differences were not statistically significant (Sup. Table 1 ).

\subsection{Concurrent endometrioid ovarian and endometrial carcinoma}

Concurrent endometrioid ovarian and endometrioid endometrial carcinoma was diagnosed in 52 patients. In 37 of these cases L1CAM was negative both in the ovarian and endometrial tumors. In 3 cases L1CAM was positive only in the endometrial tumor. In 4 cases L1CAM was positive both in the ovarian and endometrial tumors. None of the cases showed L1CAM-positivity only in the ovarian tumor. L1CAM staining was not interpretable in 5 ovarian carcinomas and in 3 endometrial carcinomas. All the patients with L1CAM positive tumors, either endometrial or ovarian, had poor outcome (Sup. Table 2). Positive L1CAM expression was strongly associated with poor overall $(p<0.0001)$ and disease-free $(p<0.0001)$ survival (Fig. 4$)$. The 5-year overall survival rate was $0 \%$ for the L1CAM-positive group and $79 \%$ for the L1CAM- 


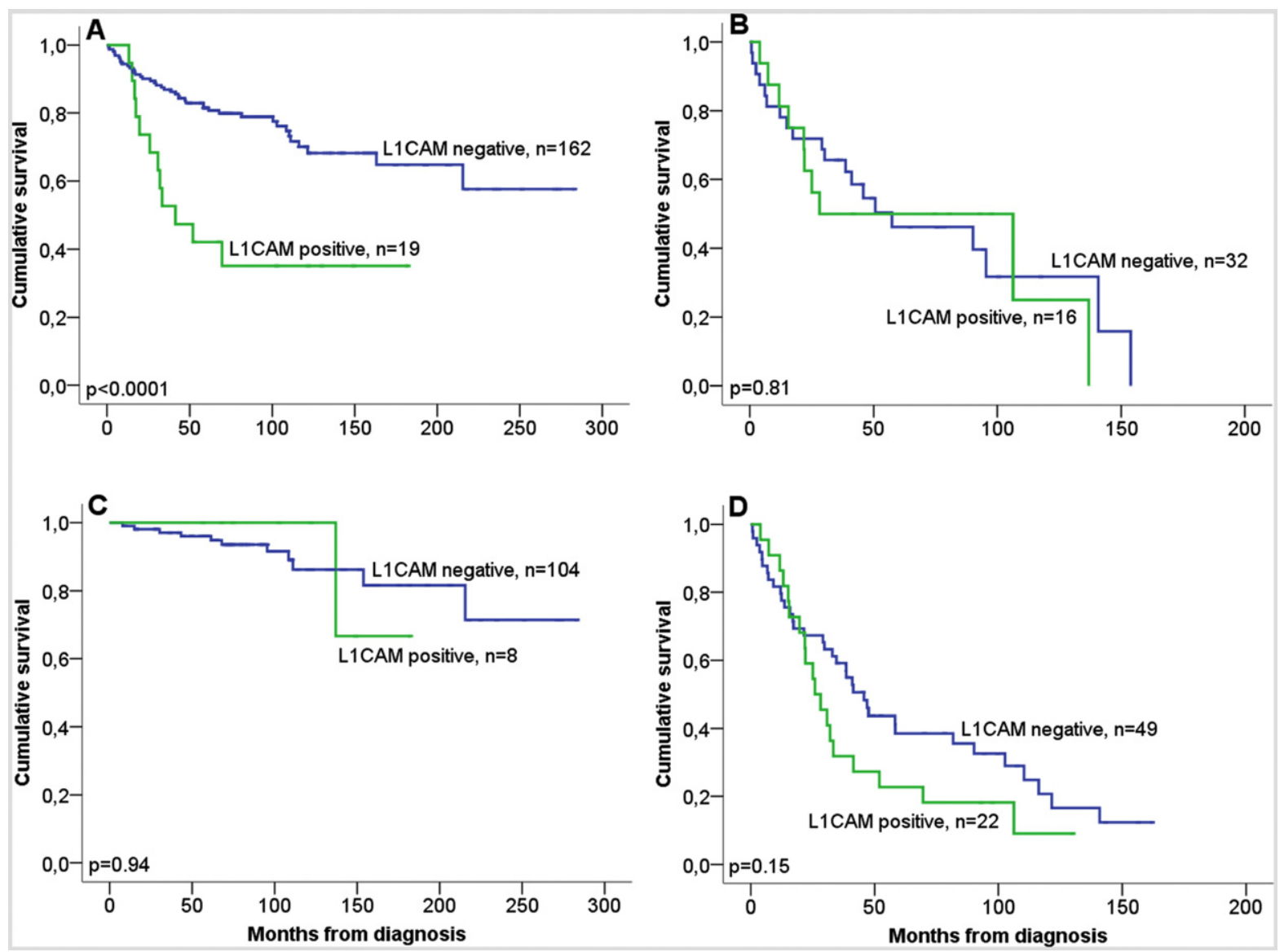

Fig. 3. Disease-specific overall survival in patients with grade 1-2 (A), grade 3 (B), stage I (C) and stage III (D) endometrioid ovarian carcinoma.

negative group. The 5 -year disease-free survival rate was $0 \%$ for the L1CAM-positive group and 76\% for the L1CAM-negative group.

\section{Discussion}

Different histological types of ovarian carcinoma are considered to be distinct diseases. However, within each histological type there is variation as regards molecular changes and biological behavior. Approximately half of the patients with endometrioid or clear cell ovarian carcinomas die of the disease. Thus there is a need for new markers to classify patients into clinically meaningful categories.
According to prevailing view both endometrioid and clear cell ovarian carcinomas have a common origin, ovarian endometriosis. Therefore it is not surprising that these carcinomas share some molecular changes in their pathogenesis e.g. ARID1A and PTEN mutations [27,28]. Some changes like microsatellite instability and $\beta$-catenin gene mutations are more typical of endometrioid carcinomas [28] and HNF1alfa mutations of clear cell carcinomas [29]. In the present study we found L1CAM expression in both endometrioid as well as clear cell carcinomas (in 15\% and $23 \%$ of cases, respectively). In endometrioid ovarian carcinoma L1CAM expression was associated with aggressive disease characteristics and poor outcome. Interestingly, those associations were not seen in the
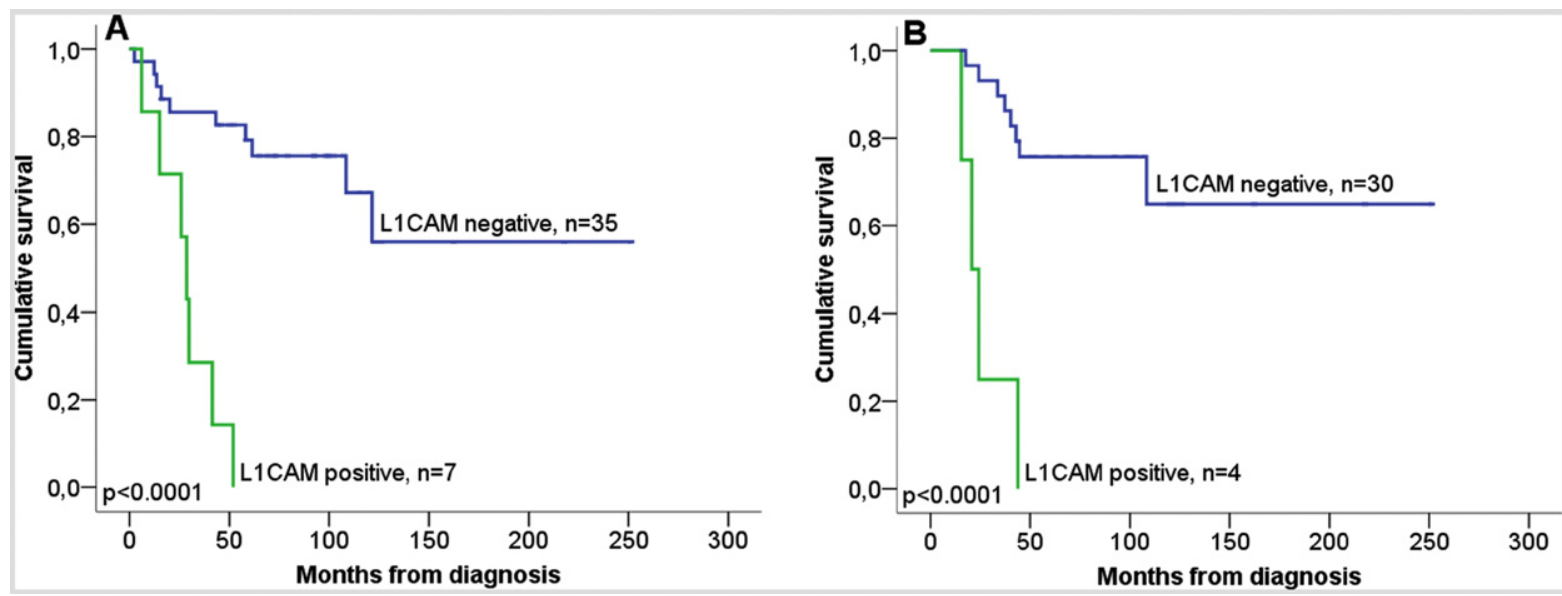

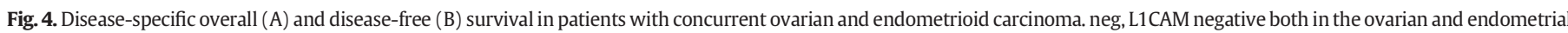
tumors; pos, L1CAM positive in the endometrial tumor and positive or negative in the ovarian tumor. 
clear cell ovarian carcinomas. This suggests a different pathogenetic role for L1CAM in endometrioid as compared to clear cell ovarian carcinomas.

In previous studies the proportion of L1CAM-positive ovarian carcinomas by immunohistochemistry was higher (43-79\%) than what we observed (15-23\%). This can be partly explained by the inclusion of mostly serous ovarian carcinomas in those studies, as the highest expression of L1CAM has been found in that particular subtype [9-11]. Also the cut-off level used to determine "positivity" was lower (L1CAM-negativity was defined as no L1CAM immunoreactivity in any section derived from the tumor) in several of these studies $[9,10]$ as compared to ours. We defined cases L1CAM positive when membranous staining of $>10 \%$ of cancer cells was present. This cut-off level was based on the findings in endometrial carcinoma, where this cut-off has led to the most optimal prediction of outcome $[12,13,16]$.

L1CAM expression was strongly associated with poor disease-specific overall and disease-free survival in endometrioid ovarian carcinomas. L1CAM expression also associated with aggressive disease characteristics such as poor differentiation and advanced stage. Previously L1CAM expression has been associated with higher grade and stage of the disease and poor outcome in ovarian carcinoma, but these studies included mostly serous carcinomas [9-11,24-26] and separate analyses concentrating on endometrioid ovarian carcinomas do not exist. Response to primary therapy has not been assessed previously. We found L1CAM expression to associate with poor response to primary therapy in endometrioid carcinomas. The association of positive L1CAM with poor overall survival appeared stronger than with incomplete response to primary treatment. This is explained by the disease free survival analysis, which was performed only for those who had complete response after primary treatment: in addition to having more often incomplete response to primary therapy, the relapse rate was also higher for those with L1CAM positive than L1CAM negative tumors.

The prognostic significance of L1CAM was further analyzed in specific clinical subgroups of endometroid ovarian carcinomas. Significant association of L1CAM expression with poor overall survival was observed in grade 1-2 carcinomas, but not in grade 3 tumors. In stage I tumors there was no difference in overall survival between L1CAM positive and negative cases. In stage III carcinomas, we observed a tendency for poorer overall survival for L1CAM-positive cases, but there was no statistically significant difference between the groups. It is possible that with increasing group size this difference would be statistically significant, so further studies are needed on this issue. Our findings suggest that L1CAM cannot be used to differentiate prognostic groups within stage I tumors, but L1CAM seems to mark biologically aggressive behavior even in morphologically well-differentiated tumors.

To our knowledge, this is the first study to investigate L1CAM expression in clear cell ovarian carcinomas. In contrast to endometrioid carcinomas, in clear cell carcinomas there was no significant association between L1CAM expression and patient survival. It has been suggested that clear cell carcinoma is composed of more than one entity with distinct morphologies, genomic changes and clinical behavior [30-33]. As there was no association with tumor stage, residual tumor size, response to therapy or patient age, L1CAM expression status does not seem to differentiate these potential subgroups. However, the number of patients with clear cell carcinoma was limited to 140 , so it is possible that increasing the group size would generate significant results as regards survival. Anyhow, the difference between survival of L1CAM negative and positive groups was much more pronounced in endometrioid than in clear cell carcinomas.

Interestingly, our findings on ovarian carcinoma were similar to those found in endometrial carcinoma. The frequency of positive expression of L1CAM has been higher in non-endometrioid than in endometrioid endometrial carcinomas [13-16,34]. Also L1CAM has been found to be a strong predictor of poor outcome in endometrioid endometrial carcinomas, but not in non-endometrioid carcinomas $[15,16]$. However, we did not find significant association between L1CAM-positivity and distant recurrences in endometrioid ovarian carcinomas as has been shown in endometrioid endometrial carcinomas [13,15,16,35]. In endometrioid ovarian carcinomas positive L1CAM expression seemed to associate with pelvic and para-aortic lymph node metastases, but the numbers of cases were too small to draw conclusions.

Our study is the first to include patients with concurrent ovarian and endometrial carcinoma. Because of the heterogeneous expression of L1CAM, it cannot be used in determining whether there are two different primary tumors or whether the ovarian tumor is a metastasis from the uterus. The L1CAM negative ovarian tumor with L1CAM positive endometrial tumor might be derived from a L1CAM negative clone in the primary endometrial carcinoma. However, all the patients with L1CAM positive tumors, either endometrial or ovarian, had poor outcome. Thus, L1CAM could be used as a prognostic marker in a clinical setting of concurrent ovarian and endometrial carcinomas.

The present study involved a large number of consecutive patients treated for endometrioid and clear cell ovarian carcinoma at the same institution. Our findings indicate that L1CAM could serve as a biomarker for predicting clinical outcome and response to therapy in patients with endometrioid ovarian carcinoma, but not in clear cell carcinomas. L1CAM may have potential as a target of therapy for a subset of endometrioid ovarian carcinomas in the future. Further studies are needed to confirm these findings and functional studies are needed to reveal the mechanistic role of L1CAM in the pathogenesis of these tumors.

Supplementary data to this article can be found online at http://dx. doi.org/10.1016/j.ygyno.2017.06.010.

\section{Conflict of interest statement}

All authors declare no conflict of interest.

\section{Acknowledgements}

We thank Gynel Arifdshan and Annikki Löfhjelm for technical assistance. This study was supported by academic funding (Helsinki University Central Hospital EVO grants TYH2015316).

\section{References}

[1] Globocan, Cancer Today. International Agency for Research on Cancer. Cancer Incidence and Mortality Worldwide, http://gco.iarc.fr/today/home 2012

[2] G.C. Stuart, First-line treatment regimens and the role of consolidation therapy in advanced ovarian cancer, Gynecol. Oncol. 90 (2003 Sep) S8-15.

[3] J.S. Berek, C. Crum, M. Friedlander, Cancer of the ovary, fallopian tube, and peritoneum, Int. J. Gynaecol. Obstet. 131 (Suppl. 2) (2015 Oct), S111-22.

[4] R.J. Kurman, I. Shih, The dualistic model of ovarian carcinogenesis: revisited, revised, and expanded, Am. J. Pathol. 186 (2016 Apr) 733-747.

[5] T. Grunewald, J.A. Ledermann, Targeted Therapies for Ovarian Cancer, Best Practice \& Research Clinical Obstetrics and Gynaecology, 2016. http://dx.doi.org/10.1016/j. bpobgyn.2016.12.001.

[6] M. Moos, R. Tacke, H. Scherer, D. Teplow, K. Fruh, M. Schachner, Neural adhesion molecule $\mathrm{L} 1$ as a member of the immunoglobulin superfamily with binding domains similar to fibronectin, Nature 334 (1988 Aug 25) 701-703.

[7] M. Schachner, Neural recognition molecules and synaptic plasticity, Curr. Opin. Cell Biol. 9 (1997 Oct) 627-634.

[8] H. Kamiguchi, M.L. Hlavin, V. Lemmon, Role of L1 in neural development: what the knockouts tell us, Mol. Cell. Neurosci. 12 (1998 Sep) 48-55.

[9] M. Fogel, P. Gutwein, S. Mechtersheimer, S. Riedle, A. Stoeck, A. Smirnov, et al., L1 expression as a predictor of progression and survival in patients with uterine and ovarian carcinomas, Lancet 362 (2003 Sep 13) 869-875.

[10] S. Zecchini, M. Bianchi, N. Colombo, R. Fasani, G. Goisis, C. Casadio, et al., The differential role of L1 in ovarian carcinoma and normal ovarian surface epithelium, Cancer Res. 68 (2008 Feb 15) 1110-1118.

[11] S. Abdel Azim, M. Duggan-Peer, S. Sprung, D. Reimer, H. Fiegl, A. Soleiman, et al., Clinical impact of L1CAM expression measured on the transcriptome level in ovarian cancer, Oncotarget 11 (2016 May).

[12] A.G. Zeimet, D. Reimer, M. Huszar, B. Winterhoff, U. Puistola, S.A. Azim, et al., L1CAM in early-stage type I endometrial cancer: results of a large multicenter evaluation, J. Natl. Cancer Inst 105 (2013 Aug 7) 1142-1150.

[13] T. Bosse, R.A. Nout, E. Stelloo, E. Dreef, H.W. Nijman, I.M. Jurgenliemk-Schulz, et al., L1 cell adhesion molecule is a strong predictor for distant recurrence and overall survival in early stage endometrial cancer: pooled PORTEC trial results, Eur. J. Cancer 50 (2014 Oct) 2602-2610.

[14] T.H. Dellinger, D.D. Smith, C. Ouyang, C.D. Warden, J.C. Williams, E.S. Han, L1CAM is an independent predictor of poor survival in endometrial cancer - an analysis of The Cancer Genome Atlas (TCGA), Gynecol. Oncol. 141 (2016 May) 336-340. 
[15] L.J. van der Putten, N.C. Visser, K. van de Vijver, M. Santacana, P. Bronsert, J. Bulten, et al., L1CAM expression in endometrial carcinomas: an ENITEC collaboration study, Br. J. Cancer (2016 Aug 9).

[16] A. Pasanen, T. Tuomi, J. Isola, S. Staff, R. Butzow, M. Loukovaara, L1 cell adhesion molecule as a predictor of disease-specific survival and patterns of relapse in endometrial cancer, Int. J. Gynecol. Cancer 26 (2016 Oct) 1465-1471.

[17] M. Huszar, G. Moldenhauer, V. Gschwend, A. Ben-Arie, P. Altevogt, M. Fogel, Expression profile analysis in multiple human tumors identifies L1 (CD171) as a molecular marker for differential diagnosis and targeted therapy, Hum. Pathol. 37 (2006 Aug) $1000-1008$

[18] J.T. Kaifi, U. Reichelt, A. Quaas, P.G. Schurr, R. Wachowiak, E.F. Yekebas, et al., L1 is associated with micrometastatic spread and poor outcome in colorectal cancer, Mod. Pathol. 20 (2007 Nov) 1183-1190.

[19] D.L. Chen, Z.L. Zeng, J. Yang, C. Ren, D.S. Wang, W.J. Wu, et al., L1cam promotes tumor progression and metastasis and is an independent unfavorable prognostic factor in gastric cancer, J. Hematol. Oncol. 6 (2013 Jun 27), 43-8722-6-43.

[20] Y.Y. Wang, L. Li, Z.S. Zhao, Y.X. Wang, Z.Y. Ye, H.Q. Tao, L1 and epithelial cell adhesion molecules associated with gastric cancer progression and prognosis in examination of specimens from 601 patients, J. Exp. Clin. Cancer Res. 32 (2013 Sep 16), 66-9966-32-66.

[21] C. Schroder, U. Schumacher, M. Fogel, F. Feuerhake, V. Muller, R.M. Wirtz, et al., Expression and prognostic value of L1-CAM in breast cancer, Oncol. Rep. 22 (2009 Nov) 1109-1117.

[22] K. Doberstein, K. Milde-Langosch, N.P. Bretz, U. Schirmer, A. Harari, I. Witzel, et al., L1CAM is expressed in triple-negative breast cancers and is inversely correlated with androgen receptor, BMC Cancer 14 (2014 Dec 15), 958-2407-14-958.

[23] J.L. Hecht, G.L. Mutter, Molecular and pathologic aspects of endometrial carcinogenesis, J. Clin. Oncol. 24 (2006 Oct 10) 4783-4791.

[24] S. Bondong, H. Kiefel, T. Hielscher, A.G. Zeimet, R. Zeillinger, D. Pils, et al., Prognostic significance of L1CAM in ovarian cancer and its role in constitutive NF-kappaB activation, Ann. Oncol. 23 (2012 Jul) 1795-1802.

[25] B. Aktas, S. Kasimir-Bauer, P. Wimberger, R. Kimmig, M. Heubner, Utility of mesothelin, L1CAM and Afamin as biomarkers in primary ovarian cancer, Anticancer Res. 33 (2013 Jan) 329-336.

[26] S. Inaguma, Z. Wang, J.P. Lasota, M.M. Miettinen, Expression of neural cell adhesion molecule L1 (CD171) in neuroectodermal and other tumors. An immunohistochemica study of 5155 tumors and critical evaluation of CD171 prognostic value in gastrointestinal stromal tumors, Oncotarget (2016 Jul 11).

[27] P.S. Munksgaard, J. Blaakaer, The association between endometriosis and ovarian cancer: a review of histological, genetic and molecular alterations, Gynecol. Oncol. 124 (2012 Jan) 164-169.

[28] J.J. Wei, J. William, S. Bulun, Endometriosis and ovarian cancer: a review of clinical, pathologic, and molecular aspects, Int. J. Gynecol. Pathol. 30 (2011 Nov) 553-568.

[29] A. Tsuchiya, M. Sakamoto, J. Yasuda, M. Chuma, T. Ohta, M. Ohki, et al., Expression profiling in ovarian clear cell carcinoma: identification of hepatocyte nuclear factor-1 beta as a molecular marker and a possible molecular target for therapy of ovarian clear cell carcinoma, Am. J. Pathol. 163 (2003 Dec) 2503-2512.

[30] E. Veras, T.L. Mao, A. Ayhan, S. Ueda, H. Lai, M. Hayran, et al., Cystic and adenofibromatous clear cell carcinomas of the ovary: distinctive tumors that differ in their pathogenesis and behavior: a clinicopathologic analysis of 122 cases, Am. J. Surg. Pathol. 33 (2009 Jun) 844-853.

[31] S. Yamamoto, H. Tsuda, H. Shimazaki, M. Takano, T. Yoshikawa, K. Kuzuya, et al. Clear cell adenocarcinoma with a component of poorly differentiated histology: a poor prognostic subgroup of ovarian clear cell adenocarcinoma, Int. J. Gynecol Pathol. 30 (2011 Sep) 431-441.

[32] Y. Uehara, K. Oda, Y. Ikeda, T. Koso, S. Tsuji, S. Yamamoto, et al., Integrated copy number and expression analysis identifies profiles of whole-arm chromosomal alterations and subgroups with favorable outcome in ovarian clear cell carcinomas, PLoS One 10 (2015 Jun 4), e0128066.

[33] D.S. Tan, M. Iravani, W.G. McCluggage, M.B. Lambros, F. Milanezi, A. Mackay, et al. Genomic analysis reveals the molecular heterogeneity of ovarian clear cell carcinomas, Clin. Cancer Res. 17 (2011 Mar 15) 1521-1534.

[34] Y.P. Geels, J.M. Pijnenborg, B.B. Gordon, M. Fogel, P. Altevogt, R. Masadah, et al., L1CAM expression is related to non-endometrioid histology, and prognostic for poor outcome in endometrioid endometrial carcinoma, Pathol. Oncol. Res. (2016 Feb 18).

[35] E. Smogeli, B. Davidson, M. Cvancarova, A. Holth, B. Katz, B. Risberg, et al., L1CAM as a prognostic marker in stage I endometrial cancer: a validation study, BMC Cancer 16 (2016 Aug 4), 596-016-2631-4. 